\title{
A novel and efficient synthesis of trivalent 9,10-dihydro-9-oxa-10- phosphaphenanthrene-10-oxide derivatives
}

\author{
Johannes Artner, Michael Ciesielski, Martin Ahlmann, Olaf Walter, and Manfred Döring* \\ Karlsruhe Research Center GmbH, Institute for Technical Chemistry, 76021 Karlsruhe, \\ Germany \\ E-mail: manfred.doering@itc-cpv.fzk.de
}

Dedicated to Prof. Ernst Anders on the occasion of his $65^{\text {th }}$ birthday

\begin{abstract}
9,10-Dihydro-9-oxa-10-phosphaphenanthrene-10-oxide (DOPO) reacts under acidic conditions with orthoformates and alkyl alcohols to form 10-alkoxy-10H-9-oxa-10-phosphaphenanthrenes (1) in high yields. 10-Dialkoxymethyl-9-oxa-10-phosphaphenanthrene-10-oxides (2) were identified as side products and synthesized as main products by the modification of the reaction conditions.
\end{abstract}

Keywords: 9,10-Dihydro-9-oxa-10-phosphaphenanthrene-10-oxide, DOPO derivatives, phosphonous acid diester, flame retardancy

\section{Introduction}

Phosphorus is capable of forming stable compounds with a coordination number of 1 to 6 and an oxidation state of - III to + V. Consequently, organophosphorus compounds are characterized by an unusually large variety of structures and a wide range of uses. They are commonly found in applications for plant protection, pharmaceutics, and flame retardancy. ${ }^{1}$

With increasing use of natural and synthetic polymeric materials and changing legislation regarding their halogen-based alternatives, phosphorus-based flame retardants are gaining more and more attention. In particular, commercially available 9,10-dihydro-9-oxa-10phosphaphenanthrene-10-oxide and its derivatives were found to exhibit outstanding performance in various polymers. ${ }^{2}$

Commonly, its derivatives are synthesized via nucleophilic reactions of DOPO on the one hand and activated double bounds (e.g. acrylics $^{3}$ ), epoxides ${ }^{4}$, halides ${ }^{5}$, and carbonyls ${ }^{6}$ on the other. Besides the increasing numbers of applications of DOPO derivatives, limitations of the 
above reactions lead to a high demand for new possibilities to incorporate this compound in larger chemical frameworks.

Here, a novel and effective synthetic approach to trivalent DOPO-based phosphonous acid diesters (1) shall be presented. Generally, diesters of phosphonous acid are formed by halophosphines reacting with alcohols in the presence of bases, or by phosphorous acid diester chlorides reacting with Grignard reagents ${ }^{1}$. Transformations of pentavalent phosphinic acid monoesters and phosphonic acid mono- or diesters into the corresponding trivalent phosphonous acid diesters and phosphites respectively, have been accomplished so far only by using silyl, germanyl, and stannyl chlorides ${ }^{7}$ and hexamethyldisilazane ${ }^{8}$. Phosphonous acid diesters and phosphites readily undergo transesterification and can be converted into their pentavalent analogs via Michaelis-Arbuzow rearrangement ${ }^{9}$.

So far, compounds (1) have only been synthesized from already trivalent 10-chloro-10H-9oxa-10-phosphaphenanthrene $\mathrm{e}^{10,11}$.

\section{Results and Discussion}

Under acidic conditions, DOPO reacts with trialkyl orthoformates and a large excess of alkyl alcohols to form trivalent 10-alkoxy-10H-9-oxa-10-phosphaphenanthrenes (1) (scheme 1, table 1). $\mathrm{R}$ in the orthoformate and $\mathrm{R}$ in the alcohol may be identical, but do not have to be. In this case - under the condition of acidic catalysis -, transesterification occurs at the product as well as at the orthoformate ${ }^{12}$. Hence, the nature of the product is defined nearly completely by the alcohol used. The structure of (1b) was revealed by X-ray diffractometry (figure 1, table 2).<smiles>O=[PH]1Oc2ccccc2-c2ccccc21</smiles>

DOPO

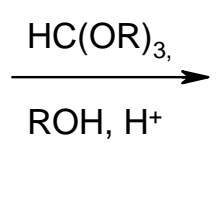

$$
\begin{aligned}
& \mathrm{R}=\mathrm{Me}: \mathbf{( 1 \mathbf { a } )} \\
& \mathrm{R}=\mathrm{Et}:(\mathbf{1 b})
\end{aligned}
$$$$
\mathrm{R}=n-\operatorname{Pr}:(\mathbf{1 c})^{\star}
$$

Scheme 1. Synthetic pathway to 10-alkoxy-10H-9-oxa-10-phosphaphenanthrenes; *different $\mathrm{R}$ at orthoformate and alcohol used for the synthesis of (1c) (see table 1). 
Table 1. Raw yields before purification by distillation or crystallization, quantified by ${ }^{31} \mathrm{P}-\mathrm{NMR}$

\begin{tabular}{cccccc}
\hline Orthoformate & Solvent & Acid & \multicolumn{2}{c}{ Products } & DOPO \\
\hline $\mathrm{HC}(\mathrm{OMe})_{3}$ & $\mathrm{MeOH}$ & $\mathrm{HCl}$ & $\mathbf{( 1 a ) : ~} 77 \%$ & $10 \% \mathrm{a}$ & $13 \%$ \\
$\mathrm{HC}(\mathrm{OEt})_{3}$ & $\mathrm{EtOH}$ & $\mathrm{HCl}$ & $\mathbf{( 1 b ) : ~} 87 \%$ & $\mathbf{( 2 b ) : ~} 7 \%$ & $6 \%$ \\
$\mathrm{HC}(\mathrm{OEt})_{3}$ & $\mathrm{EtOH}$ & $\mathrm{PTSA}$ & $\mathbf{( 1 b ) : ~} 85 \%$ & $\mathbf{( 2 b ) :} 7 \%$ & $8 \%$ \\
$\mathrm{HC}(\mathrm{OEt})_{3}$ & $n$-PrOH & $\mathrm{HCl}$ & $\mathbf{( 1 c ) : ~} 67 \%$ & $\mathbf{( 2 c ) : ~} 30 \%$ & $3 \%$ \\
$\mathrm{HC}(\mathrm{OEt})_{3}$ & $n-\mathrm{PrOH}$ & $\mathrm{PTSA}$ & $\mathbf{( 1 c ) : ~} 86 \%$ & $\mathbf{( 2 c ) : ~} 10 \%$ & $2 \%$ \\
\hline
\end{tabular}

${ }^{a}$ Not isolated. ${ }^{b}$ further $2 \%$ of non-identified byproducts arising from the use of triethyl orthoformate; PTSA = p-toluenesulfonic acid.

Replacing the alcoholic solvents by inert toluene, 10-dialkoxymethyl-9-oxa-10phosphaphenanthrene-10-oxides (2) - primarily by-products of the reaction - become the dominating products. This reaction carried out with triethyl orthoformate yielded $81 \%$ (2b), $8 \%$ (1b), and 11\% unreacted DOPO before purification (scheme 2). Similar reactions and a suggestion regarding the mechanism of P-dialkoxymethylation are cited in the literature ${ }^{13,14,15}$. The structure of (2b) was revealed by X-ray diffractometry. Comparing bond angles and distances of (1b) and (2b) one will notice - surprisingly considering the different nature of the Patoms surrounding - very high similarities within those structures (figure 1, table 2).

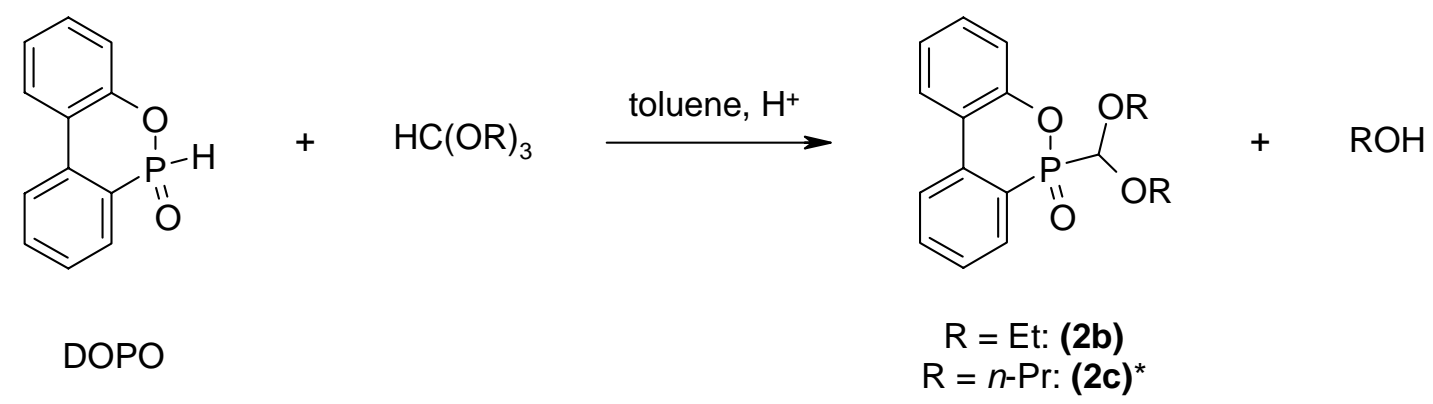

Scheme 2. Synthetic pathway to 10-dialkoxymethyl-9-oxa-10-phosphaphenanthrene-10-oxides. *isolated by-product according to scheme 1 and entry 4 in table 1 . 


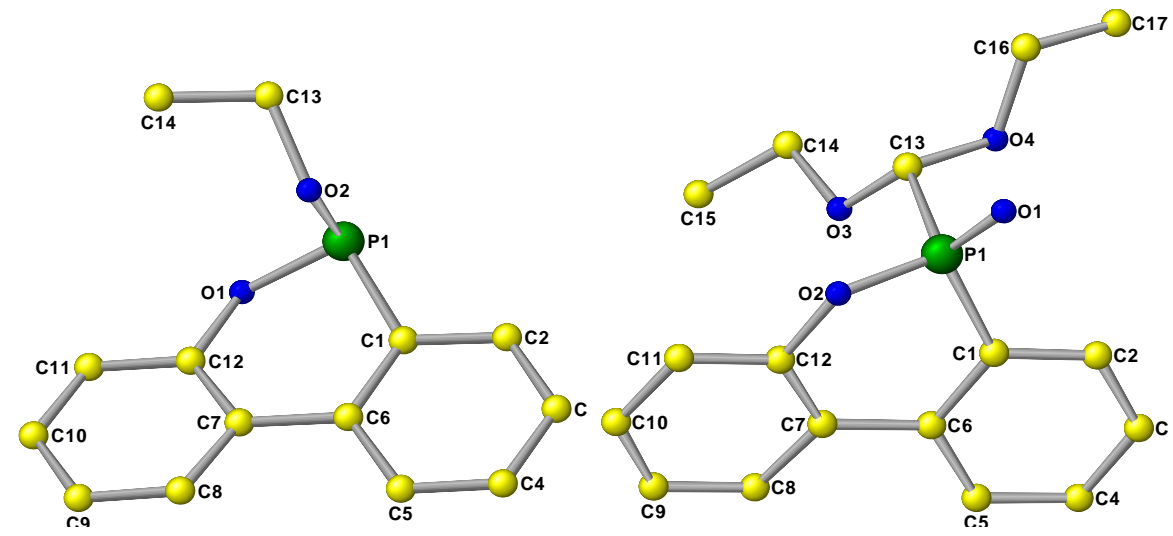

Figure 1. Molecular structures of (1b) (left) and (2b) (right) in the crystal. For reasons of clarity, one of the two molecules in the independent part of the elementary cell [(1b)], hydrogen atoms (both), and solvents [(2b)] are omitted; selected bond lengths $(\AA)$ and bond angles $\left(^{\circ}\right)$ (1b): $\mathrm{O}_{2} \mathrm{P}_{1}=1.626(4), \mathrm{O}_{1} \mathrm{P}_{1}=1.626(4), \mathrm{P}_{1} \mathrm{C}_{1}=1.809(1), \mathrm{C}_{1} \mathrm{C}_{6}=1.409(7), \mathrm{C}_{6} \mathrm{C}_{7}=1.475(6), \mathrm{C}_{7} \mathrm{C}_{12}=1.395(7)$, $\mathrm{C}_{12} \mathrm{O}_{1}=1.404(6) ; \quad \mathrm{O}_{2} \mathrm{P}_{1} \mathrm{C}_{1}=96.1(2), \quad \mathrm{O}_{2} \mathrm{P}_{1} \mathrm{O}_{1}=103.2(2), \quad \mathrm{C}_{1} \mathrm{P}_{1} \mathrm{O}_{1}=99.2(2), \quad \mathrm{P}_{1} \mathrm{O}_{1} \mathrm{C}_{12}=124.0$ (3), $\mathrm{O}_{1} \mathrm{C}_{12} \mathrm{C}_{7}=122.1(4), \mathrm{C}_{12} \mathrm{C}_{7} \mathrm{C}_{6}=120.9(4), \mathrm{C}_{7} \mathrm{C}_{6} \mathrm{C}_{1}=121.0(4), \mathrm{C}_{6} \mathrm{C}_{1} \mathrm{P}_{1}=121.7(4)$ (2b): $\mathrm{O}_{1} \mathrm{P}_{1}=1.469(2)$, $\mathrm{O}_{2} \mathrm{P}_{1}=1.593(2), \quad \mathrm{C}_{13} \mathrm{P}_{1}=1.829(2), \quad \mathrm{C}_{1} \mathrm{P}_{1}=1.779(2), \quad \mathrm{O}_{2} \mathrm{C}_{12}=1.400(2), \quad \mathrm{C}_{12} \mathrm{C}_{7}=1.402(3)$, $\mathrm{C}_{7} \mathrm{C}_{6}=1.480(3), \quad \mathrm{C}_{6} \mathrm{C}_{1}=1.407(3) ; \quad \mathrm{C}_{13} \mathrm{P}_{1} \mathrm{O}_{1}=110.4(1), \quad \mathrm{C}_{13} \mathrm{P}_{1} \mathrm{C}_{1}=108.0(1), \quad \mathrm{O}_{2} \mathrm{P}_{1} \mathrm{O}_{1}=112.3(1)$, $\mathrm{O}_{2} \mathrm{P}_{1} \mathrm{C}_{13}=105.2(1), \mathrm{P}_{1} \mathrm{C}_{1} \mathrm{C}_{6}=120.0(2), \mathrm{C}_{1} \mathrm{C}_{6} \mathrm{C}_{7}=120.9(2), \mathrm{C}_{6} \mathrm{C}_{7} \mathrm{C}_{12}=121.6(2), \mathrm{C}_{7} \mathrm{C}_{12} \mathrm{O}_{2}=122.1(2)$, $\mathrm{C}_{12} \mathrm{O}_{2} \mathrm{P}_{1}=123.0(2), \mathrm{C}_{1} \mathrm{P}_{1} \mathrm{O}_{2}=103.3(1)$.

The specific acid used ( $\mathrm{HCl}$ and p-toluenesulfonic acid were used for this work) never appeared to have any significant influence on the distribution of the products in the reactions mentioned above. The unusual yields for the fourth reaction in Table 1 are attributed to the considerably higher temperature applied $\left(\approx 100^{\circ} \mathrm{C}\right)$. As DOPO never - not even with a large excess of orthoformate - was consumed completely, a state of equilibrium is postulated.

We could not extend the reaction according to Scheme 1 to other phosphinic acid monoesters (phenylphosphinic acid ethylester), phosphonic acid diesters (phosphonic acid diethylester and methylester, cyclic 5,5-dimethyl-[1,3,2]dioxaphosphinane-2-oxide ${ }^{16}$ ), and secondary phosphine oxides (2,8-dimethylphenoxaphosphine-10-oxide $\left.{ }^{17}\right)$. Only DOPO with its special phosphacyclic ring system was capable of undergoing this transformation. When comparing bond angles at the phosphorus atom of $\mathrm{DOPO}^{18}\left(\angle \mathrm{OPO}_{\mathrm{Ph}}=112.2^{\circ} ; \angle \mathrm{OPC}=116.1^{\circ} ; \angle \mathrm{O}_{\mathrm{Ph}} \mathrm{PC}=105.3^{\circ}\right)$ with a similar non-cyclic system $\mathrm{MeOP}(\mathrm{O}) \mathrm{HAr}^{19} \quad\left(\angle \mathrm{OPO}_{\mathrm{Me}}=115.2, \quad 116.4^{\circ} ; \quad \angle \mathrm{OPC}=112.0, \quad 112.3^{\circ}\right.$; $\angle \mathrm{O}_{\mathrm{Me}} \mathrm{PC}=99.3,100.4^{\circ}$ ), ring strain as the reason of this uniqueness appears to be rather unlikely.

The key explanation may be the surprisingly low angle of torsion between the two aromatics of the DOPO ring system $\left(\angle{ }_{\text {tor }} \mathrm{C}_{\mathrm{O}} \mathrm{CCC}_{\mathrm{P}}=2.7^{\circ}\right)$ in comparison to those of (1b) $\left(\angle{ }_{\text {tor }} \mathrm{C}_{\mathrm{O}} \mathrm{CCC}_{\mathrm{P}}=11.2\right.$, $\left.11.3^{\circ}\right)$ and (2b) $\left(\angle{ }_{\text {tor }} \mathrm{C}_{\mathrm{O}} \mathrm{CCC}_{\mathrm{P}}=10.0^{\circ}\right)$. This angle is within the range of angles of torsion in the two aromatics, which indicates a more aromatic character of the phosphacyclic ring and, hence, chemical properties different from those that one might expect. Those may arise from strain 
between hydrogen atoms on $\mathrm{C}-\mathrm{C}-\mathrm{C}_{\mathrm{P}}$ and $\mathrm{C}-\mathrm{C}-\mathrm{C}_{\mathrm{O}}$, which are most closely to each other due to the low angle of torsion of the two aromatic rings. Bearing in mind the facile ring opening of DOPO with water ${ }^{20}$, a mechanism involving an initial analogous reaction of DOPO with the alcohol followed by orthoformate-enforced acid-catalyzed dehydration of the intermediate is proposed (scheme 3).

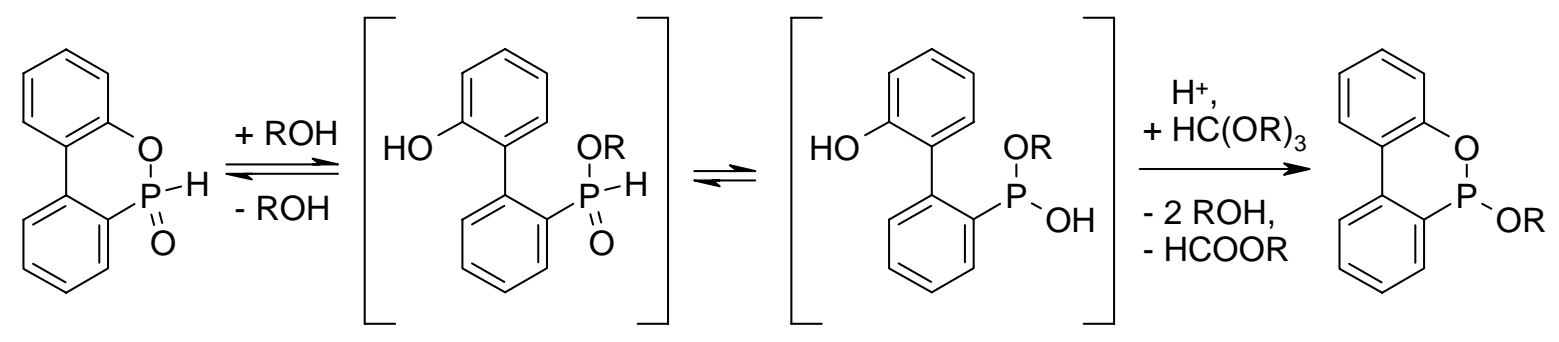

Scheme 3. Proposed reaction mechanism for the transformation of DOPO into its trivalent analogs.

Compounds (1) may be transesterificated easily with alkyl alcohols and the resulting intermediates transformed into their pentavalent analogs via Michaelis-Arbuzow rearrangement. Data and proceedings on this subject will be published in the near future.

\section{Experimental Section}

General Procedures. Infrared spectra (IR) were obtained using a Perkin-Elmer System 2000 FT-IR spectrophotometer. Nuclear magnetic resonance (NMR) spectra were recorded with a Bruker AC-250 using trimethylsilane and trimethylphosphate ${ }^{21}$ as internal standards. Elemental analysis was performed with a Vario EL III from Elementar Analysensysteme GmbH. Highresolution mass spectrometry (HRMS) and electron impact mass spectrometry (MS) were performed with a Finnigan MAT-4000-1. Melting points were obtained with a Büchi B-545 at a heating rate of $3{ }^{\circ} \mathrm{C} / \mathrm{min}$. Purity of the compounds was determind by ${ }^{1} \mathrm{H}$ - and ${ }^{31} \mathrm{P}-\mathrm{NMR}$.

For the crystallographic details of the single-crystal X-ray diffraction measurements, see Table 2. The X-ray analyses were performed using a Siemens SMART CCD 1000 diffractometer with an irradiation time of $10 \mathrm{~s}$ to $20 \mathrm{~s}$ per frame, thus collecting a full sphere of data using an $\omega$ scan technique with $\Delta \omega$ ranging from 0.3 to $0.45^{\circ}$. For searches relating to single-crystal X-ray diffraction data, the Cambridge Structural Database was used ${ }^{22}$. Data were corrected for polarization and Lorentz effects, and an experimental absorption correction was performed with SADABS ${ }^{23}$. SHELX- $97^{24}$ was used for the calculation and graphical evaluation and visualization of the data processed with $\mathrm{XPMA}^{25}$ and WinRay ${ }^{26}$. 
DOPO was provided by Schill \& Seilacher AG, Germany. Trimethyl and triethyl orthoformate, p-toluenesulfonic acid, and the solvents were purchased from Aldrich and used as received.

\section{0-Methoxy-10H-9-oxa-10-phosphaphenanthrene (1a)}

DOPO $(287.5 \mathrm{~g}, 1.33 \mathrm{~mol})$ and $2.5 \mathrm{ml} \mathrm{HCl}_{\text {conc }}$ were refluxed in $1200 \mathrm{ml}$ methanol at $85^{\circ} \mathrm{C}$ and an overpressure of $\approx 250 \mathrm{mbar}$ for $45 \mathrm{~min}$. Another $0.5 \mathrm{ml} \mathrm{HCl}_{\text {conc }}$ were added and afterwards, trimethyl orthoformate $(295 \mathrm{ml}, 2.7 \mathrm{~mol})$ was added in a dropwise manner over $5 \mathrm{~h}$. While adding the orthoformate, another $0.5 \mathrm{ml} \mathrm{HCl}_{\text {conc }}$ were added every $30 \mathrm{~min}$. Removal of volatile compounds and purification of the product $\left(\approx 0.1 \mathrm{mbar}, 130-135^{\circ} \mathrm{C}\right)$ were performed by vacuum distillation to give a clear, oily liquid with a purity exceeding $98 \%$ that very slowly solidified. Yield: $223.8 \mathrm{~g}$ (73\% of theory). $\mathrm{Mp} 42^{\circ} \mathrm{C}$. Anal $_{\text {found }}$ C, 67.97; H, 4.64. Anal $\mathrm{c}_{\text {calc }}\left(\mathrm{C}_{13} \mathrm{H}_{11} \mathrm{O}_{2} \mathrm{P}\right)$ : $\mathrm{C}$, 67.83; H, 4.82. HRMS: $\mathrm{M}_{\text {found }}=230.0516, \mathrm{M}_{\text {calc }}=230.0497$. $\mathrm{MS}(70 \mathrm{eV}): \mathrm{m} / \mathrm{z}=230,216,215$, 199, 168, 152, 139. IR (film): 1603, 1592, 1582, 1476, 1445, 1429, 1278, 1236, 1202, 1116 , $1023 \mathrm{~cm}^{-1} .{ }^{1} \mathrm{H}-\mathrm{NMR}\left(\mathrm{CDCl}_{3}\right): \delta \mathrm{H}=3.48\left(\mathrm{~d}, 3 \mathrm{H}, \mathrm{J}_{\mathrm{PH}}=11.4 \mathrm{~Hz}, \mathrm{CH}_{3}\right), 7.20\left(\mathrm{dd}, 1 \mathrm{H}, \mathrm{J}_{\mathrm{HH}} \approx 7.5\right.$, $7.5 \mathrm{~Hz}, \mathrm{O}-\mathrm{C}-\mathrm{C}-\mathrm{CH}-\mathrm{CH}), 7.27$ (d, 1H, $\left.\mathrm{J}_{\mathrm{HH}}=7.8 \mathrm{~Hz}, \mathrm{O}-\mathrm{C}-\mathrm{CH}\right), 7.36$ (dd, 1H, $\mathrm{J}_{\mathrm{HH}} \approx 7.6,7.6 \mathrm{~Hz}, \mathrm{O}-\mathrm{C}-$ $\mathrm{CH}-\mathrm{CH}), 7.47$ (dd, 1H, $\left.\mathrm{J}_{\mathrm{HH}} \approx 7.3,7.3 \mathrm{~Hz}, \mathrm{P}-\mathrm{C}-\mathrm{CH}-\mathrm{CH}\right), 7.61$ (dd, 1H, J $\mathrm{JH}_{\mathrm{H}} \approx 7.6,7.6 \mathrm{~Hz}, \mathrm{P}-\mathrm{C}-\mathrm{C}-\mathrm{CH}-$ $\mathrm{CH}), 7.71\left(\mathrm{dd}, 1 \mathrm{H}, \mathrm{J}_{\mathrm{PH}}=10.8 \mathrm{~Hz}, \mathrm{~J}_{\mathrm{HH}}=7.8 \mathrm{~Hz}, \mathrm{P}-\mathrm{C}-\mathrm{CH}\right), 7.97\left(\mathrm{~d}, 1 \mathrm{H}, \mathrm{J}_{\mathrm{HH}}=7.3 \mathrm{~Hz}, \mathrm{O}-\mathrm{C}-\mathrm{C}-\mathrm{CH}\right), 8.00$ $\left(\mathrm{d}, 1 \mathrm{H}, \mathrm{J}_{\mathrm{HH}}=7.9 \mathrm{~Hz}, \mathrm{P}-\mathrm{C}-\mathrm{C}-\mathrm{CH}\right) \mathrm{ppm} .{ }^{13} \mathrm{C}-\mathrm{NMR}\left(\mathrm{CDCl}_{3}\right): \delta \mathrm{C}=54.8\left(\mathrm{~J}_{\mathrm{PC}}=10.3 \mathrm{~Hz}, \mathrm{CH}_{3}\right), 120.3$ (O-C-CH), 122.3 ( $\left.\mathrm{J}_{\mathrm{PC}}=6.1 \mathrm{~Hz}, \mathrm{O}-\mathrm{C}-\mathrm{C}\right), 123.18$ (O-C-C-CH-CH), 123.22 (P-C-C-CH), 124.7 (O$\mathrm{C}-\mathrm{C}-\mathrm{CH}), 127.5\left(\mathrm{~J}_{\mathrm{PC}}=13.2 \mathrm{~Hz}, \mathrm{P}-\mathrm{C}-\mathrm{CH}-\mathrm{CH}\right), 129.6(\mathrm{O}-\mathrm{C}-\mathrm{CH}-\mathrm{CH}), 131.3\left(\mathrm{~J}_{\mathrm{PC}}=47.9 \mathrm{~Hz}, \mathrm{P}-\mathrm{C}-\mathrm{CH}\right)$, $131.66(\mathrm{P}-\mathrm{C}-\mathrm{C}-\mathrm{CH}-\mathrm{CH}), 131.72\left(\mathrm{~J}_{\mathrm{PC}}=17.4 \mathrm{~Hz}, \mathrm{P}-\mathrm{C} \wedge \mathrm{P}-\mathrm{C}-\mathrm{C}\right), 131.9\left(\mathrm{~J}_{\mathrm{PC}}=2.8 \mathrm{~Hz}, \mathrm{P}-\mathrm{C} \wedge \mathrm{P}-\mathrm{C}-\mathrm{C}\right)$, $149.6\left(\mathrm{~J}_{\mathrm{PC}}=9.2 \mathrm{~Hz}, \mathrm{O}-\mathrm{C}\right) \mathrm{ppm} .{ }^{31} \mathrm{P}-\mathrm{NMR}\left(\mathrm{CDCl}_{3}\right): \delta \mathrm{P}=130.7 \mathrm{ppm}$.

\section{0-Ethoxy-10H-9-oxa-10-phosphaphenanthrene (1b)}

(a) DOPO $(43.2 \mathrm{~g}, 0.2 \mathrm{~mol})$ and $0.5 \mathrm{ml} \mathrm{HCl}_{\text {conc }}$ were refluxed in $350 \mathrm{ml}$ ethanol at $90^{\circ} \mathrm{C}$ and an overpressure of $\approx 250 \mathrm{mbar}$ for $50 \mathrm{~min}$. Another $0.1 \mathrm{ml} \mathrm{HCl}_{\text {conc }}$ were added and afterwards, triethyl orthoformate $(66.5 \mathrm{ml}, 0.4 \mathrm{~mol})$ was added in a dropwise manner over $4 \mathrm{~h}$. While adding the orthoformate, another $0.1 \mathrm{ml} \mathrm{HCl}$ conc were added every $30 \mathrm{~min}$. Removal of volatile compounds and purification of the product $\left(\approx 0.1 \mathrm{mbar}, 135-142^{\circ} \mathrm{C}\right)$ were performed by vacuum distillation to give a clear, oily liquid with a purity exceeding $98 \%$ that slowly solidified. Yield: $40.2 \mathrm{~g}$ ( $82 \%$ of theory)

(b) DOPO $(183.7 \mathrm{~g}, 0.85 \mathrm{~mol})$ and the first half of p-toluenesulfonic acid monohydrate $(0.76 \mathrm{~g}$, $4 \mathrm{mmol})$ were dissolved in $500 \mathrm{ml}$ ethanol at $50^{\circ} \mathrm{C}$. Over a period of $5 \mathrm{~h}$, triethyl orthoformate $(166 \mathrm{ml}, 1 \mathrm{~mol})$, diluted with $80 \mathrm{ml}$ ethanol, was added in a dropwise manner,while maintaining a temperature of $50^{\circ} \mathrm{C}$. After $2 \mathrm{~h}$ and $4 \mathrm{~h}$, respectively, the remaining quarters of p-toluenesulfonic acid monohydrate were added. Upon the completion of the addition, the reaction mixture was kept at $50^{\circ} \mathrm{C}$ for another hour. Removal of the volatile compounds and purification of the product $\left(\approx 0.1 \mathrm{mbar}, 135-142^{\circ} \mathrm{C}\right.$ ) were performed by vacuum distillation to give a clear, oily liquid with a purity exceeding $98 \%$ that slowly solidified. Yield: $166.2 \mathrm{~g}$ (80\% of theory). 
Mp $48^{\circ}$ C. Anal $_{\text {found }}: C, 68.72 ; \mathrm{H}$, 5.16. $\mathrm{Anal}_{\text {calc }}\left(\mathrm{C}_{14} \mathrm{H}_{13} \mathrm{O}_{2} \mathrm{P}\right)$ : C, 68.85; H, 5.37. HRMS: $\mathrm{M}_{\text {found }}=244.0658, \mathrm{M}_{\text {calc }}=244.0653 . \mathrm{MS}(70 \mathrm{eV}): \mathrm{m} / \mathrm{z}=244,216,215,199,168,152$, 139. IR (film): 1604, 1592, 1582, 1475, 1444, 1429, 1387, 1278, 1236, 1202, 1116, $1037 \mathrm{~cm}^{-1}$. ${ }^{1} \mathrm{H}-\mathrm{NMR}$ $\left(\mathrm{CDCl}_{3}\right): \delta \mathrm{H}=1.14\left(\mathrm{t}, 3 \mathrm{H}, \mathrm{J}_{\mathrm{HH}}=7.1 \mathrm{~Hz}, \mathrm{CH}_{3}\right), 3.76-3.95\left(\mathrm{~m}, 2 \mathrm{H}, \mathrm{CH}_{2}\right), 7.19\left(\mathrm{dd}, 1 \mathrm{H}, \mathrm{J}_{\mathrm{HH}} \approx 7.7\right.$, 7.7Hz, O-C-C-CH-CH), $7.24\left(\mathrm{~d}, 1 \mathrm{H}, \mathrm{J}_{\mathrm{HH}}=8.1 \mathrm{~Hz}, \mathrm{O}-\mathrm{C}-\mathrm{CH}\right), 7.36$ (dd, 1H, $\mathrm{J}_{\mathrm{HH}} \approx 7.6,7.6 \mathrm{~Hz}, \mathrm{O}-\mathrm{C}-$ $\mathrm{CH}-\mathrm{CH}), 7.46$ (dd, 1H, J $\left.\mathrm{HH}_{2} \approx .4,7.4 \mathrm{~Hz}, \mathrm{P}-\mathrm{C}-\mathrm{CH}-\mathrm{CH}\right), 7.59$ (dd, 1H, J $\mathrm{JH}_{\mathrm{H}} \approx 7.7,7.7 \mathrm{~Hz}, \mathrm{P}-\mathrm{C}-\mathrm{C}-\mathrm{CH}-$ $\mathrm{CH}), 7.70\left(\mathrm{dd}, 1 \mathrm{H}, \mathrm{J}_{\mathrm{PH}}=11.3 \mathrm{~Hz}, \mathrm{~J}_{\mathrm{HH}}=7.3 \mathrm{~Hz}, \mathrm{P}-\mathrm{C}-\mathrm{CH}\right), 7.97\left(\mathrm{~d}, 1 \mathrm{H}, \mathrm{J}_{\mathrm{HH}}=7.7 \mathrm{~Hz}, \mathrm{O}-\mathrm{C}-\mathrm{C}-\mathrm{CH}\right), 7.99$ $\left(\mathrm{d}, 1 \mathrm{H}, \mathrm{J}_{\mathrm{HH}}=7.3 \mathrm{~Hz}, \mathrm{P}-\mathrm{C}-\mathrm{C}-\mathrm{CH}\right) \mathrm{ppm} .{ }^{13} \mathrm{C}-\mathrm{NMR}\left(\mathrm{CDCl}_{3}\right): \delta \mathrm{C}=17.0\left(\mathrm{~J}_{\mathrm{PC}}=4.9 \mathrm{~Hz}, \mathrm{CH}_{3}\right), 64.4$ $\left(\mathrm{J}_{\mathrm{PC}}=12.5 \mathrm{~Hz}, \mathrm{CH}_{2}\right), 120.3(\mathrm{O}-\mathrm{C}-\mathrm{CH}), 122.5\left(\mathrm{~J}_{\mathrm{PC}}=6.1 \mathrm{~Hz}, \mathrm{O}-\mathrm{C}-\mathrm{C}\right), 123.1$ (O-C-C-CH-CH), 123.3 (P-C-C-CH), 124.7 (O-C-C-CH), 127.4 (J $\left(\mathrm{J}_{\mathrm{PC}}=48.0 \mathrm{~Hz}, \mathrm{P}-\mathrm{C}-\mathrm{CH}\right), 131.5(\mathrm{P}-\mathrm{C}-\mathrm{C}-\mathrm{CH}-\mathrm{CH}), 131.9 \quad\left(\mathrm{~J}_{\mathrm{PC}}=2.9 \mathrm{~Hz}, \mathrm{P}-\mathrm{C} \wedge \mathrm{P}-\mathrm{C}-\mathrm{C}\right), 132.2$ $\left(\mathrm{J}_{\mathrm{PC}}=15.9 \mathrm{~Hz}, \mathrm{P}-\mathrm{C} \wedge \mathrm{P}-\mathrm{C}-\mathrm{C}\right), 149.6\left(\mathrm{~J}_{\mathrm{PC}}=8.7 \mathrm{~Hz}, \mathrm{O}-\mathrm{C}\right) \mathrm{ppm} .{ }^{31} \mathrm{P}-\mathrm{NMR}\left(\mathrm{CDCl}_{3}\right): \delta \mathrm{P}=128.3 \mathrm{ppm}$.

\section{0-Diethoxymethyl-9-oxa-10-phosphaphenanthrene-10-oxide (2b)}

Triethyl orthoformate $(10.0 \mathrm{ml}, 60 \mathrm{mmol})$ and $0.2 \mathrm{ml} \mathrm{HCl}$ conc were added to a suspension of DOPO $(5.40 \mathrm{~g}, 25 \mathrm{mmol})$ in $20 \mathrm{ml}$ of toluene at $35^{\circ} \mathrm{C}$. After the suspension became clear, the temperature was raised to $50^{\circ} \mathrm{C}$ and kept for $3 \mathrm{~h}$. Volatiles were removed down to a total volume of approximately $15 \mathrm{ml}$, and the precipitating crystalline, colorless product with a purity exceeding 98\% was filtered off. Yield: $5.39 \mathrm{~g}$ (68\% of theory). Recrystallization in EtOH/ $\mathrm{H}_{2} \mathrm{O}=2: 3$ gave crystals suitable for X-ray diffractometry.

Mp $93{ }^{\circ} \mathrm{C}$. Anal found $_{\text {f }} \mathrm{C}$, 64.55; H, 6.05. $\mathrm{Anal}_{\text {calc }}\left(\mathrm{C}_{17} \mathrm{H}_{19} \mathrm{O}_{4} \mathrm{P}\right)$ : C, 64.15; H, 6.02. HRMS: $\mathrm{M}_{\text {found }}=318.1060, \mathrm{M}_{\text {calc }}=318.1021 . \mathrm{MS}(70 \mathrm{eV}): \mathrm{m} / \mathrm{z}=318,245,216,215,199,168,152,139$, 103. IR(KBr): 1591, 1584, 1560, 1476, 1446, 1428, 1384, 1302, 1275, 1243, 1216, 1104, 1061 $\mathrm{cm}^{-1} .{ }^{1} \mathrm{H}-\mathrm{NMR}\left(\mathrm{CDCl}_{3}\right): \delta \mathrm{H}=0.89\left(\mathrm{t}, 3 \mathrm{H}, \mathrm{J}_{\mathrm{HH}}=7.0 \mathrm{~Hz}, \mathrm{CH}_{3}{ }^{\prime}\right), 1.18\left(\mathrm{t}, 3 \mathrm{H}, \mathrm{J}_{\mathrm{HH}}=7.0 \mathrm{~Hz}, \mathrm{CH}_{3}{ }^{\prime}{ }^{\prime}\right)$, $3.48\left(\mathrm{qd}, 1 \mathrm{H}, \mathrm{J}_{\mathrm{HH}}=7.0,9.2 \mathrm{~Hz}, \mathrm{CH}_{\mathrm{a}}{ }^{\prime}-\mathrm{CH}_{3}{ }^{\prime}\right)$, 3.64-3.90 (m, 3H, $\mathrm{CH}_{\mathrm{b}}{ }^{\prime}-\mathrm{CH}_{3}{ }^{\prime}, \mathrm{CH}_{\mathrm{a}}{ }^{\prime}{ }^{-}-\mathrm{CH}_{3}{ }^{\prime}$,, $\mathrm{CH}_{\mathrm{b}}{ }^{\prime}$ '$\mathrm{CH}_{3}{ }^{\prime}$ ) $, 4.90\left(\mathrm{~d}, 1 \mathrm{H}, \mathrm{J}_{\mathrm{PH}}=5.9 \mathrm{~Hz}, \mathrm{P}-\mathrm{CH}\right), 7.167\left(\mathrm{~d}, 1 \mathrm{H}, \mathrm{J}_{\mathrm{HH}}=8.0 \mathrm{~Hz}, \mathrm{O}-\mathrm{C}-\mathrm{CH}\right), 7.168$ (dd, 1H, $\left.\mathrm{J}_{\mathrm{HH}} \approx 7.7,7.7 \mathrm{~Hz}, \mathrm{O}-\mathrm{C}-\mathrm{C}-\mathrm{CH}-\mathrm{CH}\right), 7.30\left(\mathrm{dd}, 1 \mathrm{H}, \mathrm{J}_{\mathrm{HH}} \approx 7.7,7.7 \mathrm{~Hz}, \mathrm{O}-\mathrm{C}-\mathrm{CH}-\mathrm{CH}\right), 7.46$ (ddd, 1H, $\left.\mathrm{J}_{\mathrm{HH}} \approx 7.5,7.5 \mathrm{~Hz}, \mathrm{~J}_{\mathrm{PH}}=3.0 \mathrm{~Hz}, \mathrm{P}-\mathrm{C}-\mathrm{CH}-\mathrm{CH}\right), 7.65\left(\mathrm{dd}, 1 \mathrm{H}, \mathrm{J}_{\mathrm{HH}} \approx 7.8,7.8 \mathrm{~Hz}, \mathrm{P}-\mathrm{C}-\mathrm{C}-\mathrm{CH}-\mathrm{CH}\right), 7.86$ $\left(\mathrm{d}, 1 \mathrm{H}, \mathrm{J}_{\mathrm{HH}}=8.2 \mathrm{~Hz}, \mathrm{O}-\mathrm{C}-\mathrm{C}-\mathrm{CH}\right), 7.92\left(\mathrm{dd}, 1 \mathrm{H}, \mathrm{J}_{\mathrm{HH}}=8.1 \mathrm{~Hz}, \mathrm{~J}_{\mathrm{PH}}=5.1 \mathrm{~Hz}, \mathrm{P}-\mathrm{C}-\mathrm{C}-\mathrm{CH}\right), 8.01$ (dd, $1 \mathrm{H}$, $\left.\mathrm{J}_{\mathrm{HH}}=7.6 \mathrm{~Hz}, \mathrm{~J}_{\mathrm{PH}}=12.3 \mathrm{~Hz}, \mathrm{P}-\mathrm{C}-\mathrm{CH}\right) \mathrm{ppm} .{ }^{13} \mathrm{C}-\mathrm{NMR}\left(\mathrm{CDCl}_{3}\right): \delta \mathrm{C}=14.4\left(\mathrm{CH}_{3}{ }^{\prime}\right), 14.8\left(\mathrm{CH}_{3}{ }^{\prime}{ }^{\prime}\right), 65.6$ $\left(\mathrm{J}_{\mathrm{PC}}=9.8 \mathrm{~Hz}, \mathrm{CH}_{2}{ }^{\prime}{ }^{\prime}\right), 65.8\left(\mathrm{~J}_{\mathrm{PC}}=10.4 \mathrm{~Hz}, \mathrm{CH}_{2}{ }^{\prime}\right), 101.4\left(\mathrm{~J}_{\mathrm{PC}}=159.0 \mathrm{~Hz}, \mathrm{P}-\mathrm{CH}\right), 119.3\left(\mathrm{~J}_{\mathrm{PC}}=6.4 \mathrm{~Hz}, \mathrm{O}-\right.$ $\mathrm{C}-\mathrm{CH}), 121.2\left(\mathrm{~J}_{\mathrm{PC}}=114.3 \mathrm{~Hz}, \mathrm{P}-\mathrm{C}\right), 121.7\left(\mathrm{~J}_{\mathrm{PC}}=9.7 \mathrm{~Hz}, \mathrm{O}-\mathrm{C}-\mathrm{C}\right), 123.0\left(\mathrm{~J}_{\mathrm{PC}}=9.7 \mathrm{~Hz}, \mathrm{P}-\mathrm{C}-\mathrm{C}-\mathrm{CH}\right)$, 123.9 (O-C-C-CH-CH), 124.5 (O-C-C-CH), 127.9 (J $\left.\mathrm{JC}_{\mathrm{PC}}=12.8 \mathrm{~Hz}, \mathrm{P}-\mathrm{C}-\mathrm{CH}-\mathrm{CH}\right), 129.9$ (O-C-CH$\mathrm{CH}), 131.7\left(\mathrm{~J}_{\mathrm{PC}}=10.0 \mathrm{~Hz}, \mathrm{P}-\mathrm{C}-\mathrm{CH}\right), 133.4\left(\mathrm{~J}_{\mathrm{PC}}=2.3 \mathrm{~Hz}, \mathrm{P}-\mathrm{C}-\mathrm{C}-\mathrm{CH}-\mathrm{CH}\right), 136.5\left(\mathrm{~J}_{\mathrm{PC}}=6.2 \mathrm{~Hz}, \mathrm{P}-\mathrm{C}-\right.$ C), $149.9\left(\mathrm{~J}_{\mathrm{PC}}=9.0 \mathrm{~Hz}, \mathrm{O}-\mathrm{C}\right) \mathrm{ppm} .{ }^{31} \mathrm{P}-\mathrm{NMR}\left(\mathrm{CDCl}_{3}\right): \delta \mathrm{P}=26.1 \mathrm{ppm}$.

10-n-Propoxy-10H-9-oxa-10-phosphaphenanthrene (1c), 10-di-n-propoxymethyl-9-oxa-10phospha- phenanthrene-10-oxide (2c)

(a) DOPO $(59.4 \mathrm{~g}, 0.275 \mathrm{~mol})$ and $0.6 \mathrm{ml} \mathrm{HCl}$ conc were refluxed in $600 \mathrm{ml} 1$-propanol for $60 \mathrm{~min}$. Another $0.1 \mathrm{ml}$ of $\mathrm{HCl}_{\text {conc }}$ were added and afterwards, triethyl orthoformate $(92 \mathrm{ml}, 0.55 \mathrm{~mol})$ was added in a dropwise manner over $3 \mathrm{~h}$. While adding the orthoformate, another $0.1 \mathrm{ml} \mathrm{HCl}_{\text {conc }}$ were added every $30 \mathrm{~min}$. Volatile compounds were removed by slowly raising the temperature up to 
$120^{\circ} \mathrm{C}$ and reducing the pressure down to $10 \mathrm{mbar}$ during $3 \mathrm{~h}$ (whereas ethanol was removed faster from equilibrium than 1-propanol). Separation/purification of the products ((1c): $\approx 0.1 \mathrm{mbar}, 136-140^{\circ} \mathrm{C}$, purity: $98+\%$; (2c): $\approx 0.01 \mathrm{mbar}, 179-184^{\circ} \mathrm{C}$, purity $95+\%$ ) were performed by vacuum distillation to give clear, oily liquids. Yield: (1c): $44.0 \mathrm{~g}$ (62\% of theory); (2c): $27.1 \mathrm{~g}$ (28\% of theory).

(b) DOPO (162.1g, 0.75mol) and two thirds of p-toluenesulfonic acid monohydrate $(0.67 \mathrm{~g}, 3.5$ $\mathrm{mmol}$ ) were dissolved in $420 \mathrm{ml} \mathrm{1-propanol} \mathrm{at} 50^{\circ} \mathrm{C}$. Over a period of $7 \mathrm{~h}$, triethyl orthoformate (166ml, 1mol), diluted with $80 \mathrm{ml}$ 1-propanol, was added in a dropwise manner, while maintaining a temperature of $50^{\circ} \mathrm{C}$. After $3.5 \mathrm{~h}$ of adding the remaining third of p-toluenesulfonic acid monohydrate was added. Upon the completion of the addition, the reaction mixture was kept at $50^{\circ} \mathrm{C}$ for another hour. Volatile compounds were removed by slowly raising the temperature up to $120^{\circ} \mathrm{C}$ and reducing the pressure down to $10 \mathrm{mbar}$ during $3 \mathrm{~h}$ (whereas ethanol was removed faster from equilibrium than 1-propanol). Purification of the product $\left(\approx 0.1 \mathrm{mbar}, 136-140^{\circ} \mathrm{C}\right)$ was performed by vacuum distillation to give a clear, oily liquid with a purity exceeding $98 \%$. Yield: $152.7 \mathrm{~g}$ (79\% of theory) (1c).

(1c) Anal $\mathrm{A}_{\text {found }}: \mathrm{C}, 69.49 ; \mathrm{H}$, 5.69. $\mathrm{Anal}_{\text {calc }}\left(\mathrm{C}_{15} \mathrm{H}_{15} \mathrm{O}_{2} \mathrm{P}\right): \mathrm{C}, 69.76 ; \mathrm{H}$, 5.85. HRMS: $\mathrm{M}_{\text {found }}=258.0808, \mathrm{M}_{\text {calc }}=258.0810$. MS $(70 \mathrm{eV}): \mathrm{m} / \mathrm{z}=258,216,215,199,168,152$, 139. IR (film): 1604, 1593, 1582, 1475, 1445, 1429, 1389, 1277, 1235, 1202, 1116, $1061 \mathrm{~cm}^{-1}$. ${ }^{1} \mathrm{H}-\mathrm{NMR}$ $\left(\mathrm{CDCl}_{3}\right): \delta \mathrm{H}=0.74\left(\mathrm{t}, 3 \mathrm{H}, \mathrm{J}_{\mathrm{HH}}=7.4 \mathrm{~Hz}, \mathrm{CH}_{3}\right), 1.50\left(\mathrm{qt}, 2 \mathrm{H}, \mathrm{J}_{\mathrm{HH}} \approx 6.7,6.7 \mathrm{~Hz}, \mathrm{CH}_{2}-\mathrm{CH}_{3}\right), 3.69-3.81$ $\left(\mathrm{m}, 2 \mathrm{H}, \mathrm{O}-\mathrm{CH}_{2}\right), 7.18\left(\mathrm{dd}, 1 \mathrm{H}, \mathrm{J}_{\mathrm{HH}} \approx 7.3,7.3 \mathrm{~Hz}, \mathrm{O}-\mathrm{C}-\mathrm{C}-\mathrm{CH}-\mathrm{CH}\right), 7.22\left(\mathrm{~d}, 1 \mathrm{H}, \mathrm{J}_{\mathrm{HH}}=7.2 \mathrm{~Hz}, \mathrm{O}-\mathrm{C}-\right.$ $\mathrm{CH}), 7.34\left(\mathrm{dd}, 1 \mathrm{H}, \mathrm{J}_{\mathrm{HH}} \approx 7.6,7.6 \mathrm{~Hz}, \mathrm{O}-\mathrm{C}-\mathrm{CH}-\mathrm{CH}\right), 7.45\left(\mathrm{dd}, 1 \mathrm{H}, \mathrm{J}_{\mathrm{HH}} \approx 7.4,7.4 \mathrm{~Hz}, \mathrm{P}-\mathrm{C}-\mathrm{CH}-\mathrm{CH}\right)$, 7.59 (dd, 1H, J $\left.\mathrm{HH}_{\mathrm{H}} \approx 7.3,7.3 \mathrm{~Hz}, \mathrm{P}-\mathrm{C}-\mathrm{C}-\mathrm{CH}-\mathrm{CH}\right), 7.69$ (dd, 1H, $\left.\mathrm{J}_{\mathrm{PH}}=11.4 \mathrm{~Hz}, \mathrm{~J}_{\mathrm{HH}}=7.4 \mathrm{~Hz}, \mathrm{P}-\mathrm{C}-\mathrm{CH}\right)$, $7.95\left(\mathrm{~d}, 1 \mathrm{H}, \mathrm{J}_{\mathrm{HH}}=7.6 \mathrm{~Hz}, \mathrm{O}-\mathrm{C}-\mathrm{C}-\mathrm{CH}\right), 7.98\left(\mathrm{~d}, 1 \mathrm{H}, \mathrm{J}_{\mathrm{HH}}=7.6 \mathrm{~Hz}, \mathrm{P}-\mathrm{C}-\mathrm{C}-\mathrm{CH}\right) \mathrm{ppm} .{ }^{13} \mathrm{C}-\mathrm{NMR}$ $\left(\mathrm{CDCl}_{3}\right): \delta \mathrm{C}=10.0\left(\mathrm{CH}_{3}\right), 24.4\left(\mathrm{~J}_{\mathrm{PC}}=4.7 \mathrm{~Hz}, \mathrm{CH}_{2}-\mathrm{CH}_{3}\right), 70.4\left(\mathrm{~J}_{\mathrm{PC}}=11.7 \mathrm{~Hz}, \mathrm{O}-\mathrm{CH}_{2}\right), 120.4(\mathrm{O}-\mathrm{C}-$ $\mathrm{CH}), 122.5$ ( $\left.\mathrm{J}_{\mathrm{PC}}=6.1 \mathrm{~Hz}, \mathrm{O}-\mathrm{C}-\mathrm{C}\right), 123.1$ (O-C-C-CH-CH), 123.3 (P-C-C-CH), 124.7 (O-C-C-CH), $127.4\left(\mathrm{~J}_{\mathrm{PC}}=13.3 \mathrm{~Hz}, \mathrm{P}-\mathrm{C}-\mathrm{CH}-\mathrm{CH}\right), 129.4(\mathrm{O}-\mathrm{C}-\mathrm{CH}-\mathrm{CH}), 131.2\left(\mathrm{~J}_{\mathrm{PC}}=48.0 \mathrm{~Hz}, \mathrm{P}-\mathrm{C}-\mathrm{CH}\right), 131.5(\mathrm{P}-$ $\mathrm{C}-\mathrm{C}-\mathrm{CH}-\mathrm{CH}), 131.9\left(\mathrm{~J}_{\mathrm{PC}}=2.8 \mathrm{~Hz}, \mathrm{P}-\mathrm{C} \wedge \mathrm{P}-\mathrm{C}-\mathrm{C}\right), 132.4\left(\mathrm{~J}_{\mathrm{PC}}=16.1 \mathrm{~Hz}, \mathrm{P}-\mathrm{C} \wedge \mathrm{P}-\mathrm{C}-\mathrm{C}\right), 149.6$ $\left(\mathrm{J}_{\mathrm{PC}}=9.0 \mathrm{~Hz}, \mathrm{O}-\mathrm{C}\right) \mathrm{ppm} .{ }^{31} \mathrm{P}-\mathrm{NMR}\left(\mathrm{CDCl}_{3}\right): \delta \mathrm{P}=128.8 \mathrm{ppm}$.

(2c) Anal found $_{\text {f }} \mathrm{C}, 66.27 ; \mathrm{H}$, 6.62. Anal $\mathrm{l}_{\text {calc }}\left(\mathrm{C}_{19} \mathrm{H}_{23} \mathrm{O}_{4} \mathrm{P}\right)$ : C, 65.89; H, 6.69. HRMS: $\mathrm{M}_{\text {found }}=346.1328, \mathrm{M}_{\text {calc }}=346.1334 . \mathrm{MS}(70 \mathrm{eV}): \mathrm{m} / \mathrm{z}=346,245,216,215,199,168,152,139$, 131. IR (film): 1595, 1584, 1559, 1477, 1448, 1298, 1275, 1236, 1208, 1149, 1109, $1067 \mathrm{~cm}^{-1}$. ${ }^{1} \mathrm{H}-\mathrm{NMR}\left(\mathrm{CDCl}_{3}\right): \delta \mathrm{H}=0.60\left(\mathrm{t}, 3 \mathrm{H}, \mathrm{J}_{\mathrm{HH}}=7.4 \mathrm{~Hz}, \mathrm{CH}_{3}{ }^{\prime}\right), 0.84\left(\mathrm{t}, 3 \mathrm{H}, \mathrm{J}_{\mathrm{HH}}=7.4 \mathrm{~Hz}, \mathrm{CH}_{3}{ }^{\prime}{ }^{\prime}\right), 1.26(\mathrm{qt}$, $\left.2 \mathrm{H}, \mathrm{J}_{\mathrm{HH}} \approx 7.0,7.0 \mathrm{~Hz}, \mathrm{CH}_{2}{ }^{\prime}-\mathrm{CH}_{3}{ }^{\prime}\right), 1.55$ (qt, $\left.2 \mathrm{H}, \mathrm{J}_{\mathrm{HH}} \approx 7.1,7.1 \mathrm{~Hz}, \mathrm{CH}_{2}{ }^{\prime}{ }^{\prime}-\mathrm{CH}_{3}{ }^{\prime}{ }^{\prime}\right), 3.35$ (td, $1 \mathrm{H}$,

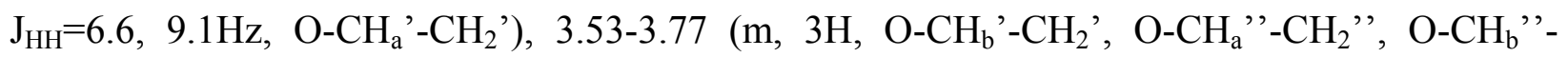
$\mathrm{CH}_{2}{ }^{\prime}$ ), $4.86\left(\mathrm{~d}, 1 \mathrm{H}, \mathrm{J}_{\mathrm{PH}}=6.3 \mathrm{~Hz}, \mathrm{P}-\mathrm{CH}\right), 7.14\left(\mathrm{~d}, 1 \mathrm{H}, \mathrm{J}_{\mathrm{HH}}=8.1 \mathrm{~Hz}, \mathrm{O}-\mathrm{C}-\mathrm{CH}\right), 7.16$ (dd, 1H, $\left.\mathrm{J}_{\mathrm{HH}} \approx 7.3,7.3 \mathrm{~Hz}, \mathrm{O}-\mathrm{C}-\mathrm{C}-\mathrm{CH}-\mathrm{CH}\right), 7.29$ (dd, 1H, $\left.\mathrm{J}_{\mathrm{HH}} \approx 7.5,7.5 \mathrm{~Hz}, \mathrm{O}-\mathrm{C}-\mathrm{CH}-\mathrm{CH}\right), 7.45$ (ddd, 1H, $\left.\mathrm{J}_{\mathrm{HH}} \approx 7.5,7.5 \mathrm{~Hz}, \mathrm{~J}_{\mathrm{PH}}=3.0 \mathrm{~Hz}, \mathrm{P}-\mathrm{C}-\mathrm{CH}-\mathrm{CH}\right), 7.65$ (dd, 1H, $\left.\mathrm{J}_{\mathrm{HH}} \approx 7.8,7.8 \mathrm{~Hz}, \mathrm{P}-\mathrm{C}-\mathrm{C}-\mathrm{CH}-\mathrm{CH}\right), 7.86$ $\left(\mathrm{d}, 1 \mathrm{H}, \mathrm{J}_{\mathrm{HH}}=8.3 \mathrm{~Hz}, \mathrm{O}-\mathrm{C}-\mathrm{C}-\mathrm{CH}\right), 7.92\left(\mathrm{dd}, 1 \mathrm{H}, \mathrm{J}_{\mathrm{HH}}=7.5 \mathrm{~Hz}, \mathrm{~J}_{\mathrm{PH}}=4.5 \mathrm{~Hz}, \mathrm{P}-\mathrm{C}-\mathrm{C}-\mathrm{CH}\right), 7.98(\mathrm{dd}, 1 \mathrm{H}$, $\left.\mathrm{J}_{\mathrm{HH}}=6.8 \mathrm{~Hz}, \mathrm{~J}_{\mathrm{PH}}=11.5 \mathrm{~Hz}, \mathrm{P}-\mathrm{C}-\mathrm{CH}\right) \mathrm{ppm} .{ }^{13} \mathrm{C}-\mathrm{NMR}\left(\mathrm{CDCl}_{3}\right): \delta \mathrm{C}=10.0\left(\mathrm{CH}_{3}{ }^{\prime}\right), 10.3\left(\mathrm{CH}_{3}{ }^{\prime}{ }^{\prime}\right), 22.5$ $\left(\mathrm{CH}_{2}{ }^{\prime}-\mathrm{CH}_{3}{ }^{\prime}\right), 22.8\left(\mathrm{CH}_{2}{ }^{\prime}{ }^{\prime}-\mathrm{CH}_{3}{ }^{\prime}{ }^{\prime}\right), 71.8\left(\mathrm{~J}_{\mathrm{PC}}=8.2 \mathrm{~Hz}, \mathrm{O}-\mathrm{CH}_{2}{ }^{\prime}\right), 72.0\left(\mathrm{~J}_{\mathrm{PC}}=7.1 \mathrm{~Hz}, \mathrm{O}-\mathrm{CH}_{2}{ }^{\prime}{ }^{\prime}\right), 102.0$ 
$\left(\mathrm{J}_{\mathrm{PC}}=158.3 \mathrm{~Hz}, \quad \mathrm{P}-\mathrm{CH}\right), \quad 119.6 \quad\left(\mathrm{~J}_{\mathrm{PC}}=6.4 \mathrm{~Hz}, \quad \mathrm{O}-\mathrm{C}-\mathrm{CH}\right), \quad 121.5 \quad\left(\mathrm{~J}_{\mathrm{PC}}=113.9 \mathrm{~Hz}, \quad \mathrm{P}-\mathrm{C}\right), \quad 121.8$ $\left(\mathrm{J}_{\mathrm{PC}}=9.8 \mathrm{~Hz}, \mathrm{O}-\mathrm{C}-\mathrm{C}\right), 123.1\left(\mathrm{~J}_{\mathrm{PC}}=9.8 \mathrm{~Hz}, \mathrm{P}-\mathrm{C}-\mathrm{C}-\mathrm{CH}\right), 124.0(\mathrm{O}-\mathrm{C}-\mathrm{C}-\mathrm{CH}-\mathrm{CH}), 124.7$ (O-C-C-CH), $128.1\left(\mathrm{~J}_{\mathrm{PC}}=12.9 \mathrm{~Hz}, \mathrm{P}-\mathrm{C}-\mathrm{CH}-\mathrm{CH}\right), 130.1(\mathrm{O}-\mathrm{C}-\mathrm{CH}-\mathrm{CH}), 132.0\left(\mathrm{~J}_{\mathrm{PC}}=9.9 \mathrm{~Hz}, \mathrm{P}-\mathrm{C}-\mathrm{CH}\right), 133.5$ $\left(\mathrm{J}_{\mathrm{PC}}=2.4 \mathrm{~Hz}, \mathrm{P}-\mathrm{C}-\mathrm{C}-\mathrm{CH}-\mathrm{CH}\right), 136.7\left(\mathrm{~J}_{\mathrm{PC}}=6.2 \mathrm{~Hz}, \mathrm{P}-\mathrm{C}-\mathrm{C}\right), 150.2\left(\mathrm{~J}_{\mathrm{PC}}=9.0 \mathrm{~Hz}, \mathrm{O}-\mathrm{C}\right) \mathrm{ppm} .{ }^{31} \mathrm{P}-\mathrm{NMR}$ $\left(\mathrm{CDCl}_{3}\right): \delta \mathrm{P}=26.1 \mathrm{ppm}$.

Table 2. Crystallographic data

\begin{tabular}{|c|c|c|}
\hline Compound & (1b) & (2b) \\
\hline Formula & $2 \mathrm{C}_{14} \mathrm{H}_{13} \mathrm{O}_{2} \mathrm{P}$ & $\mathrm{C}_{17} \mathrm{H}_{19} \mathrm{O}_{4} \mathrm{P} * 0.5 \mathrm{H}_{2} \mathrm{O}$ \\
\hline$M_{\mathrm{r}}$ & 488.43 & 327.30 \\
\hline$T(\mathrm{~K})$ & $200(2)$ & $200(2)$ \\
\hline$\lambda(\operatorname{Mo~K} \alpha)(\AA)$ & 0.71073 & 0.71073 \\
\hline Crystal system & monoclinic & monoclinic \\
\hline Space group & P2(1)/n (No. 14) & $\mathrm{C} 2 / \mathrm{c}$ (No. 15) \\
\hline$a(\AA)$ & $18.524(3)$ & $21.5460(15)$ \\
\hline$b(\AA)$ & $7.5153(11)$ & $8.3490(6)$ \\
\hline$c(\AA)$ & 19.021(3) & $19.9173(14)$ \\
\hline$\alpha\left({ }^{\circ}\right)$ & 90 & 90 \\
\hline$\beta\left({ }^{\circ}\right)$ & $111.162(2)$ & $114.1450(10)$ \\
\hline$\gamma\left({ }^{\circ}\right)$ & 90 & 90 \\
\hline$V(\AA)$ & $2469.3(6)$ & $3269.4(4)$ \\
\hline $\mathrm{Z}$ & 4 & 8 \\
\hline$D_{\text {calc }}\left(\mathrm{g} \mathrm{cm}^{-3}\right)$ & 1.314 & 1.330 \\
\hline$\mu(\operatorname{Mo~K\alpha })\left(\mathrm{mm}^{-1}\right)$ & 0.209 & 0.187 \\
\hline$F(000)$ & 1024 & 1384 \\
\hline Crystal size $\left(\mathrm{mm}^{3}\right)$ & $0.3 \times 0.2 \times 0.3$ & $0.25 \times 0.15 \times 0.1$ \\
\hline$\theta$ Range $\left({ }^{\circ}\right)$ & $1.32-28.34$ & $2.07-28.32$ \\
\hline$h k l$ Range & $-24 / 24,-9 / 9,-25 / 25$ & $-28 / 28,-11 / 11,-26 / 26$ \\
\hline Reflections collected & 24027 & 16905 \\
\hline Unique reflections $\left(R_{\text {int }}\right)$ & $6011(0.0650)$ & $4018(0.0583)$ \\
\hline Reflections observed $(\mathrm{I}>2 \sigma)$ & 4349 & 2599 \\
\hline Parameters refined & 317 & 218 \\
\hline Goodness-of-fit on $\mathrm{F}^{2}$ & 1.070 & 1.038 \\
\hline$(\Delta p)_{\max , \min }\left(\mathrm{e} \AA^{-3}\right)$ & $1.784,-0.934$ & $0.528,-0.389$ \\
\hline Final $R$ indices $(\mathrm{I}>2 \sigma)$ & $\begin{array}{c}R_{1}=0.1086 \\
w R_{2}=0.3089\end{array}$ & $\begin{array}{c}R_{1}=0.0519 \\
w R_{2}=0.1311\end{array}$ \\
\hline$R$ indices (all data) & $\begin{array}{c}R_{1}=0.1340 \\
w R_{2}=0.3272 \\
\end{array}$ & $\begin{array}{c}R_{1}=0.0919 \\
w R_{2}=0.1481 \\
\end{array}$ \\
\hline
\end{tabular}




\section{Supplementary Information}

Crystallographic data of the structures were deposited at the Cambridge Crystallographic Database Centre, supplementary publications Nos. CCDC 624397 (1b) and 624396 (2b). Copies of this information may be obtained free of charge from: The Director, CCDC, 12 Union Road, Cambridge CB2 1EZ, UK (Fax: +44-1223-336033, e-mail: deposit@ccdc.cam.ac.uk or www: http://www.ccdc.cam.ac.uk).

\section{References}

1. Svara, J.; Weferling, N.; Hofmann, T. Organic Phosphorus Compounds, Ullmann's Encyclopedia of Industrial Chemistry, online edition; Wiley-VCH; 2002.

2. Lu, S.-Y.; Hamerton I. Prog. Polym. Sci. 2002, 27, 1661.

3. Saito, T.; Ohishi, H. U.S. Patent 4.280.951, 1981.

4. Bauer, M. Eur. Pat. Appl. 1,544,227 A1, 2005.

5. Beletskaya, I.; Neganova, E.; Veits, Y. Russian J. Org. Chem. 2004, 40, 1782.

6. Liu, Y.-L.; Wu, C.-S.; Hsu, K.-Y.; Chang, T.-C. J. Polym. Sci., Part A: Polym. Chem. 2002, 40, 2329.

7. Issleib, K.; Walther B. Angew. Chem. 1967, 79, 59.

8. Lebedev, E.; Pudovik, A.; Tsyganov, B.; Nazmutdinov, R.; Romanov, G. Zh. Obs. Khim. 1977, 47, 765.

9. Bhattacharya, A.; Thyagarajan, G. Chem. Rev. 1981, 81, 415.

10. Kleiner, H.-J. Eur. Pat. Appl. 787738 A1, 1997.

11. Chernyshev, E.; Aksenov, V.; Ponomarev, V.; Golubtsow, S.; Bugerenko, E. Zh. Obs. Khim. 1972, 42, 93.

12. Busch, A. J. Am. Chem. Soc. 1952, 74, 554.

13. Costisella, B.; Gross, H. J. Prakt. Chem. 1974, 316, 550.

14. Gallagher, M.; Honegger, H. Tetrahedron Lett. 1977, 34, 2987.

15. Costisella, B.; Gross, H. J. Prakt. Chem. 1979, 321, 361.

16. Maffei, M.; Buono, G. Tetrahedron 2003, 59, 8821.

17. Granoth, I.; Kalir, A.; Pelah Z. Isr. J. Chem. 1968, 6, 651.

18. Schäfer, A.; Seibold, S.; Walter, O.; Döring, M. J. Appl. Polym. Sci., submitted. CCDC 617871.

19. compound 3 (two molecules in the independent part of the elementary cell) In Kilian, P.; Slawin, A.; Woollins, J. Inorg. Chim. Acta 2005, 358, 1719.

20. Saito, T. U.S. Patent 3.702.878, 1972.

21. Streck, R.; Barnes, A. J. Spectrochimica Acta Part A 1999, 55, 1049.

22. Allen, F. H.; Kennard, O. Chemical Design Automation News 1993, 8, 31.

23. Siemens area detector absorption correction programme, Siemens, 1997. 
24. Sheldrick, G. M. Universität Göttingen, 1997.

25. Zsolnai, L.; Huttner, G. Universität Heidelberg, 1994.

26. Soltek, R. Universtität Heidelberg, 1997. 